\title{
Tubular aggregate myopathy caused by a novel mutation in the cytoplasmic domain of STIM1
}

\section{OPEN}

Hidehiko Okuma, MD

Fumiaki Saito, MD, PhD

Jun Mitsui, MD, PhD

Yuji Hara, PhD

Yuki Hatanaka, MD

Miki Ikeda, MT

Teruo Shimizu, MD,

$\mathrm{PhD}$

Kiichiro Matsumura,

$\mathrm{MD}, \mathrm{PhD}$

Jun Shimizu, MD, PhD

Shoji Tsuji, MD, PhD

Masahiro Sonoo, MD,

$\mathrm{PhD}$

Correspondence to

Dr. Saito:

f-saito@med.teikyo-u.ac.jp

\section{ABSTRACT}

Objective: To identify the gene mutation of tubular aggregate myopathy (TAM) and gain mechanistic insight into the pathogenesis of the disorder.

Methods: We described a family affected by autosomal dominant TAM and performed exome and Sanger sequencing to identify mutations. We further analyzed the functional significance of the identified mutation by expression studies and intracellular $\mathrm{Ca}^{2+}$ measurements.

Results: A 42-year-old man presented with slowly progressive muscle weakness and atrophy in all 4 limbs and the trunk. Muscle biopsy and microscopic examination revealed tubular aggregates in his skeletal muscle. Genetic analysis of this family identified a novel heterozygous mutation, c.1450_1451insGA (p.lle484ArgfsX21), in stromal interaction molecule 1 (STIM1), a Ca ${ }^{2+}$ sensor in sarcoplasmic reticulum. We transfected cultured cells with STIM1 and demonstrated that the mutant STIM1 exhibited aggregation-like appearance in shrunk cytoplasm. Furthermore, we revealed that the intracellular $\mathrm{Ca}^{2+}$ influx is decreased by the mutant STIM1.

Conclusions: The novel mutation p.lle484ArgfsX21 is located in the cytoplasmic C-terminal inhibitory domain (CTID) of STIM1. However, all mutations reported so far in TAM reside in the luminal N-terminal EF hand region. The aggregation-like appearance of STIM1 and the decreased intracellular $\mathrm{Ca}^{2+}$ influx in cells transfected with CTID mutant are in sharp contrast to these previous reports. Taken together, these findings indicate that mutations of STIM1 cause TAM through the dysregulation of $\mathrm{Ca}^{2+}$ homeostasis. Neurol Genet 2016;2:e50; doi: 10.1212/ NXG.0000000000000050

\section{GLOSSARY}

CTID = C-terminal inhibitory domain; H\&E = hematoxylin and eosin; $\mathbf{m R N A}=$ messenger RNA; RT = reverse transcriptase; SOCE $=$ store-operated $\mathrm{Ca}^{2+}$ entry; $\mathbf{S R}=$ sarcoplasmic reticulum; STIM1 = stromal interaction molecule 1; TAM = tubular aggregate myopathy; TR = tetrazolium reductase.

Tubular aggregate myopathy (TAM) is a disorder characterized by slowly progressive muscle weakness and atrophy. The pathologic feature is the appearance of tubular aggregates in skeletal muscle. The tubular aggregates are a subsarcolemmal accumulation of granular materials that is stained bright purple with Gomori trichrome staining and exhibits accumulation of regular arrays of membrane tubules under an electron microscope. ${ }^{1}$ The tubular aggregates are thought to originate from sarcoplasmic reticulum $(\mathrm{SR})^{2}$; however, the exact mechanism leading to their formation and the significance on muscle fibers remain unknown. ${ }^{3}$

Recently, several mutations in stromal interaction molecule 1 (STIM1), a $\mathrm{Ca}^{2+}$ sensor in SR, have been identified in dominantly inherited TAM (OMIM \#160565). ${ }^{4-6}$ All mutations demonstrated so far have been missense mutations located in the EF hand of the $\mathrm{N}$-terminal luminal region and are thought to disrupt the $\mathrm{Ca}^{2+}$ binding to this region. In this article, we present a

From the Department of Neurology (H.O., F.S., Y. Hatanaka, M.I., K.M., M.S.), School of Medicine, and Department of Sport and Medical Science (T.S.), Faculty of Medical Technology, Teikyo University, Tokyo, Japan; Department of Neurology (J.M., J.S., S.T.), Graduate School of Medicine, The University of Tokyo, Tokyo, Japan; and Department of Synthetic Chemistry and Biological Chemistry (Y. Hara), Graduate School of Engineering, Kyoto University, Kyoto, Japan.

Funding information and disclosures are provided at the end of the article. Go to Neurology.org/ng for full disclosure forms. The Article Processing Charge was paid by the authors.

This is an open access article distributed under the terms of the Creative Commons Attribution-NonCommercial-NoDerivatives License 4.0 (CC BY-NC-ND), which permits downloading and sharing the work provided it is properly cited. The work cannot be changed in any way or used commercially. 
dominant TAM family in whom a novel insertion mutation was identified in the C-terminal inhibitory domain (CTID) located in the cytoplasmic region of STIM1 and investigate the functional significance of the mutation using cultured cells.

METHODS Standard protocol approvals, registrations, and patient consents. This study was approved by the institutional review board of Teikyo University. The patients gave written informed consent to participate in this study.

Histologic analyses. Muscle specimens were taken from the biceps brachii muscle. Standard techniques were used for the preparation of frozen sections; hematoxylin and eosin (H\&E), Gomori trichrome, NADH-tetrazolium reductase (TR), and ATPase staining; and immunofluorescent and electron microscopic analysis. Mouse monoclonal antibody against STIM1 and rabbit polyclonal antibody against ORAI were obtained from BD Biosciences (Franklin Lakes, NJ) and Merck (Darmstadt, Germany), respectively.

Genetic analysis. Genomic DNA samples were extracted from peripheral blood leukocytes. Exonic sequences were enriched using a SureSelect v5+UTRs kit (Agilent, Santa Clara, CA) and subjected to nucleotide sequence analysis using a Hiseq2500 platform (Illumina, San Diego, CA). Burrows-Wheeler Aligner and Samtools were used with default settings for the alignment of raw reads and variant detection. The protein-altering variants in STIM1 and ORAI1 were filtered by excluding those present in 600 healthy Japanese controls. Direct nucleotide sequence analysis was performed using an ABI 3100 Genetic Analyzer (Thermo Fisher Scientific, Waltham, MA). Messenger RNA (mRNA) was extracted from muscle biopsy specimen using RNeasy Fibrous tissue mini kit (Qiagen, Hilden, Germany) and amplified by reverse transcriptase (RT)-PCR with SuperScript (Thermo Fisher Scientific).

Expression study of STIM1 in C2C12 myoblasts. Expression plasmid encoding human STIM1 complementary DNA was purchased from OriGene and the mutation c.1450_1451insGA was introduced with a KOD-Plus mutagenesis kit (Toyobo, Osaka, Japan). Two days after transfection using Effectene (Qiagen), C2C12 myoblasts were labeled with anti-STIM1, Alexa Fluor 594-conjugated phalloidin (Thermo Fisher Scientific) and 4',6diamidino-2-phenylindole (DAPI). The images were obtained using an FSX100 fluorescence microscope (Olympus, Tokyo, Japan). Statistical differences were evaluated by the $t$ test.

Measurement of changes in intracellular $\mathrm{Ca}^{2+}$ concentration. $\mathrm{Ca}^{2+}$ measurements were carried out as previously described ${ }^{7}$ with minor modifications. HEK293 cells were cotransfected with plasmids expressing STIM1 and pIRES2mCherry. Transfection was carried out using X-tremeGENE HP transfection reagent (Roche, Basel, Switzerland). Transfected HEK293 cells were trypsinized and seeded onto poly-L-lysine-coated glass coverslips the next day. $\mathrm{Ca}^{2+}$ imaging was performed $42-48$ hours after transfection. HEK293 cells on coverslips were loaded with Fura-2 by incubation in Dulbecco's modified Eagle medium containing $5 \mu \mathrm{M}$ Fura-2/AM (Dojindo, Kumamoto, Japan) at $37^{\circ} \mathrm{C}$ for 40 minutes. The coverslips were then placed in a perfusion chamber mounted on the stage of a microscope (Axioobserver Z1; Carl Zeiss, Oberkochen, Germany). Transfected cells were identified by the detection of fluorescence from mCherry. Fura-2 fluorescence images recorded at 10-second intervals were analyzed using Physiology software (Carl Zeiss).
RESULTS Clinical data. A 42-year-old Japanese man presented with difficulty walking. He first noticed slight difficulty walking when he was 37 years old. At the age of 40 , running and climbing stairs became difficult. His mother also had difficulty walking and was in a wheelchair when she was 40 years old. His parents were not consanguineous. On neurologic examination, pupils and ocular movement were normal. The temporalis and masseter muscles on both sides were atrophic. Muscle atrophy and weakness was evident in all 4 limbs, and the bilateral triceps surae muscles were most prominently affected (figure 1A). The paraspinal muscles were also atrophic and he had winged scapulae (figure 1B). His gait was lordotic. Ankle joint contracture was observed bilaterally. He did not exhibit myalgia, cramps, scoliosis, rigid spine, or symptoms suggesting Stormorken syndrome, including miosis, thrombocytopenia, asplenia, or ichthyosis. ${ }^{8} \mathrm{He}$ had no history of repetitive infections indicative of immunodeficiency.9 Blood cell counts and routine blood biochemistry were normal, except serum creatine kinase was elevated to 798 IU/L (normal range <150). Needle EMG showed chronic myopathic changes. Muscle MRI revealed disseminated high-intensity areas on T1-weighted images in the quadriceps femoris, triceps surae, and paraspinal muscles, indicating fatty replacement in these muscles (figure 1C).

Pathologic findings. H\&E staining demonstrated increased variability of muscle fiber diameter and occasional centrally nucleated myofibers. In addition, many fibers showed irregularly shaped dark red staining (figure 1D). These dark red accumulations were stained intense blue by NADH-TR (figure 1E) and bright purple with Gomori trichrome staining (figure $1 F)$, indicating that these are tubular aggregates. A serial section stained with ATPase $(\mathrm{pH}$ 4.6) further demonstrated that the tubular aggregates were in type 2 fibers (figure 1G). Electron microscopic analysis revealed that some areas in the myofiber were replaced by numerous tubular structures (figure $1 \mathrm{H}$ ) and that each tubule was arranged in a honeycomb-like structure that showed double-walled membranes at higher magnification (figure 1H, inset).

Genetic analysis and localization of STIM1 in skeletal muscle. The clinical and microscopic findings characteristic of TAM prompted us to perform genetic analysis. Exome sequence analysis and subsequent Sanger sequencing of the proband revealed a novel heterozygous insertion mutation, c.1450_1451insGA (p.Ile484ArgfsX21), in exon 7 of STIM1 (figure 2A). The same mutation was identified in his mother but not in healthy family members (figure 2B). The amino acid 484 resides in the CTID located in the cytoplasmic region of STIM1 (figure 2C). To assess the localization of STIM1 in the skeletal muscle of the patient, we 

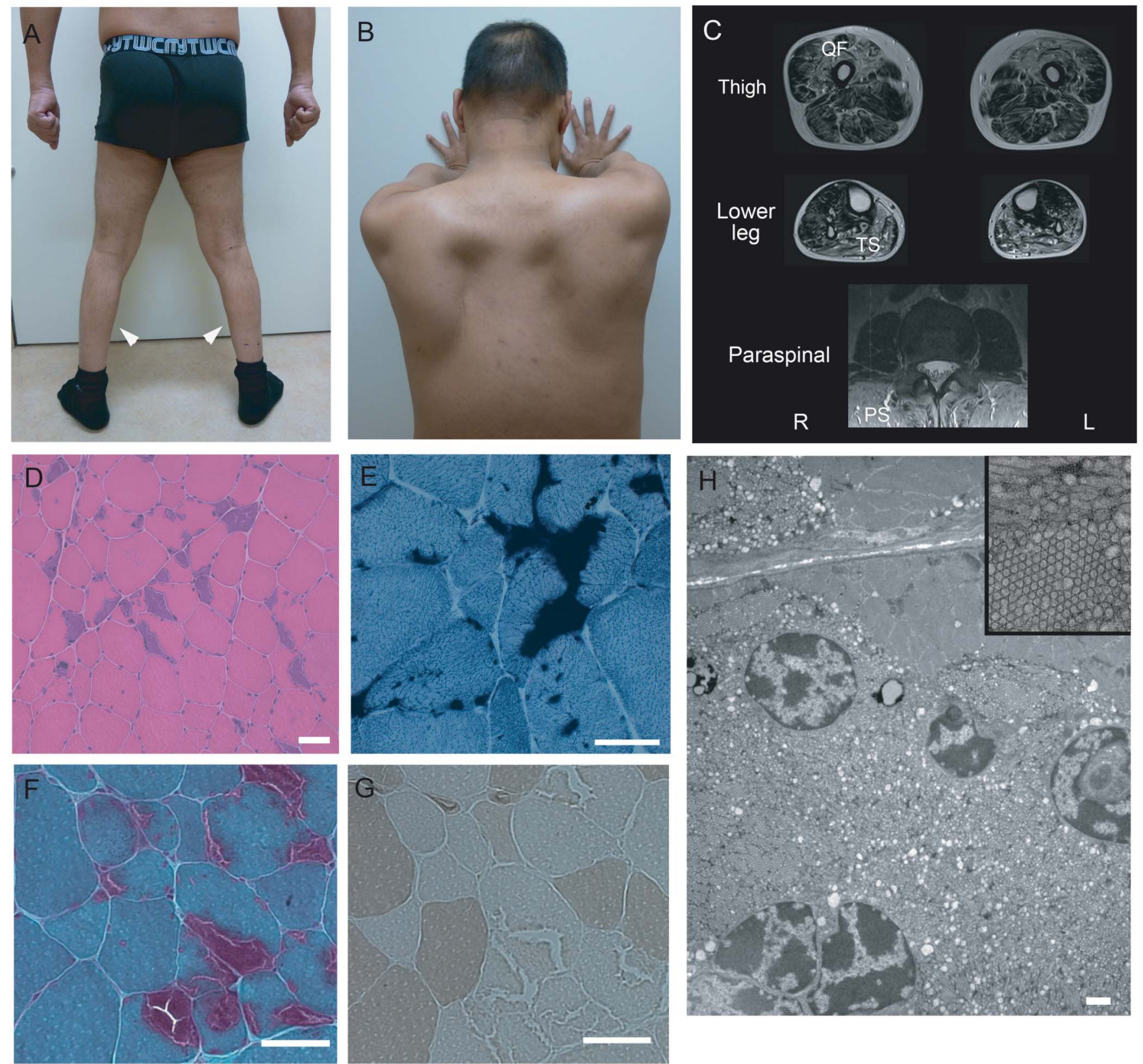

(A) Muscle atrophy is evident in all 4 limbs. Among them, the triceps surae muscle (white arrowhead) is most prominently affected. (B) The patient exhibits winged scapulae due to atrophy of the paraspinal muscles. (C) Muscle MRI shows high-intensity areas on T1-weighted images in the quadriceps femoris, triceps surae, and paraspinal muscles, indicating fatty replacement in these muscles. (D) Hematoxylin and eosin (H\&E) staining reveals the increased variability of muscle fiber diameter and occasional centrally nucleated fibers. Many fibers show tubular aggregates that are stained dark red by H\&E (D), intense blue by NADH-tetrazolium reductase (E), and bright purple with Gomori trichrome staining (F). (G) A serial section stained with ATPase (pH 4.6) demonstrates that tubular aggregates are in type 2 fibers. Bar $=50 \mu \mathrm{m}$. $(\mathrm{H})$ Electron microscopy shows that some areas in the myofiber are replaced by numerous tubular structures. At higher magnification, each tubule is arranged in a honeycomb-like structure showing double-walled membranes (inset). Bar $=1 \mu \mathrm{m} . \mathrm{PS}=$ paraspinal muscles; $\mathrm{QF}=$ quadriceps femoris; $\mathrm{TS}=$ triceps surae.

performed immunofluorescent microscopic analysis. The immunolabeling of STIM1 and ORAI1, a calcium channel in plasma membrane, colocalized to the tubular aggregates visualized by Gomori trichrome staining (figure 2D), which is consistent with previous reports. ${ }^{4,7}$ Because premature termination codons often result in nonsense-mediated decay of mRNA, we analyzed mRNA transcript in the muscle biopsy specimen.
Sequencing of the RT-PCR product demonstrated that both wild-type and the c.1450_1451insGA mutant transcripts are present (figure 2E).

Expression of mutant STIM1 in C2C12 myoblasts. To investigate the functional significance of the c.1450_1451insGA mutation, we transfected $\mathrm{C} 2 \mathrm{C} 12$ myoblasts with wild-type and mutant STIM1. Typically, wild-type 
Figure 2 Genetic analysis and expression of STIM1 in the skeletal muscle of a patient with tubular aggregate myopathy

A

$G$ G $A$ A $A T T G T G T C$
$G A T T G T G$

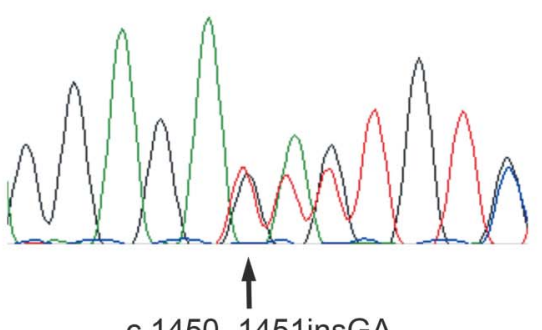

c.1450_1451insGA
B

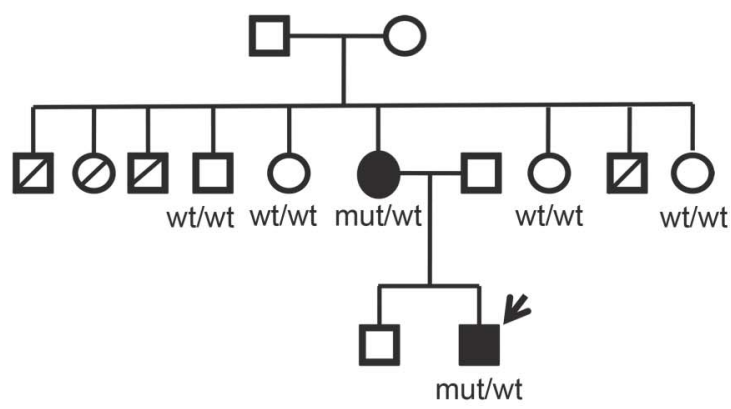

C

His109Asn/Arg
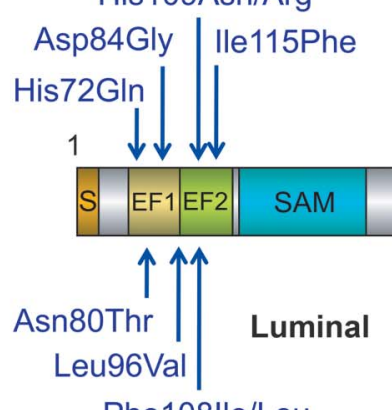

Phe108lle/Leu
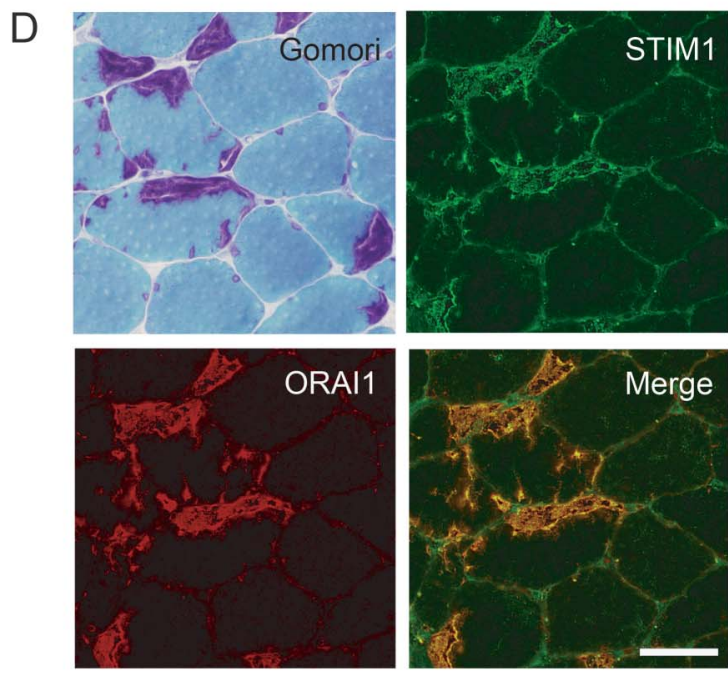

$\mathrm{E}$

Cytoplasmic
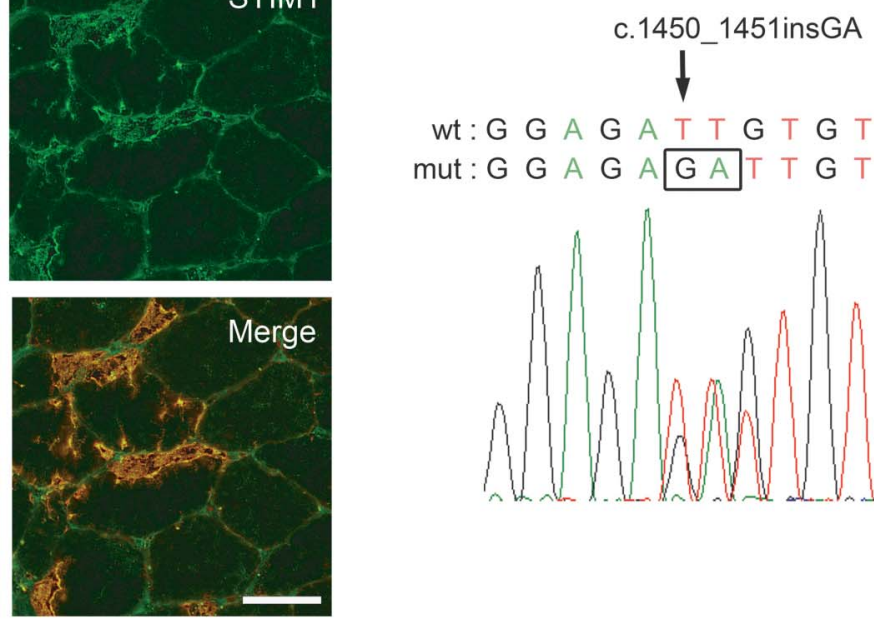

wt: G G A G A T T G G T C mut: $G$ G A G A $A$ A T $T$ T G

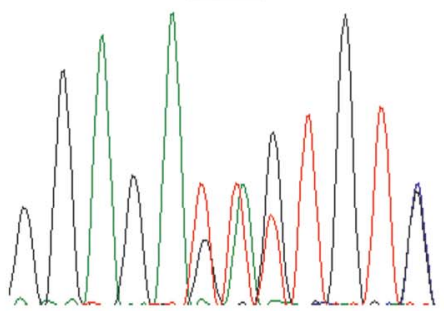

(A) Sanger sequencing reveals a heterozygous insertion mutation, c.1450_1451insGA, in STIM1. (B) Pedigree of the family indicates dominant inheritance and sequence analysis demonstrates the segregation of the heterozygous mutations with the disease. (C) Schematic representation of the domain structure of STIM1. The mutation p.lle484ArgfsX21 resides in the C-terminal inhibitory domain (CTID), which is located in the cytoplasmic region of STIM1 (red type). All missense mutations reported so far reside in the EF hand in the luminal region (blue type). (D) Serial sections of Gomori trichrome staining and immunofluorescent images indicate that both STIM1 and ORAI1 colocalize to the tubular aggregates. Bar $=50 \mu \mathrm{m}$. (E) Sanger sequencing of reverse transcriptase-PCR product amplified from muscle biopsy specimen demonstrates that both wild-type and mutant transcripts are present. $\mathrm{CC} 1=$ coiled-coil region $1 ; \mathrm{K}=$ polylysine; $\mathrm{S}=$ signal peptide; $\mathrm{SAM}=$ sterile $\alpha$ motif; SORE = STIM1 Orai activating region; SP = serine- and proline-rich domain; TM = transmembrane domain.

STIM1 displayed diffuse distribution in $\mathrm{C} 2 \mathrm{C} 12$ cells (figure 3A). In sharp contrast, mutant STIM1 concentrated intensely around nuclei and exhibited an aggregation-like appearance in shrunk cytoplasm (figure 3A). Counting cells with the diffuse vs aggregation-like localization of STIM1 confirmed that the majority of cells expressing wild-type STIM1 showed diffuse localization whereas most cells expressing mutant STIM1 exhibited an aggregationlike pattern (figure 3B). 
A
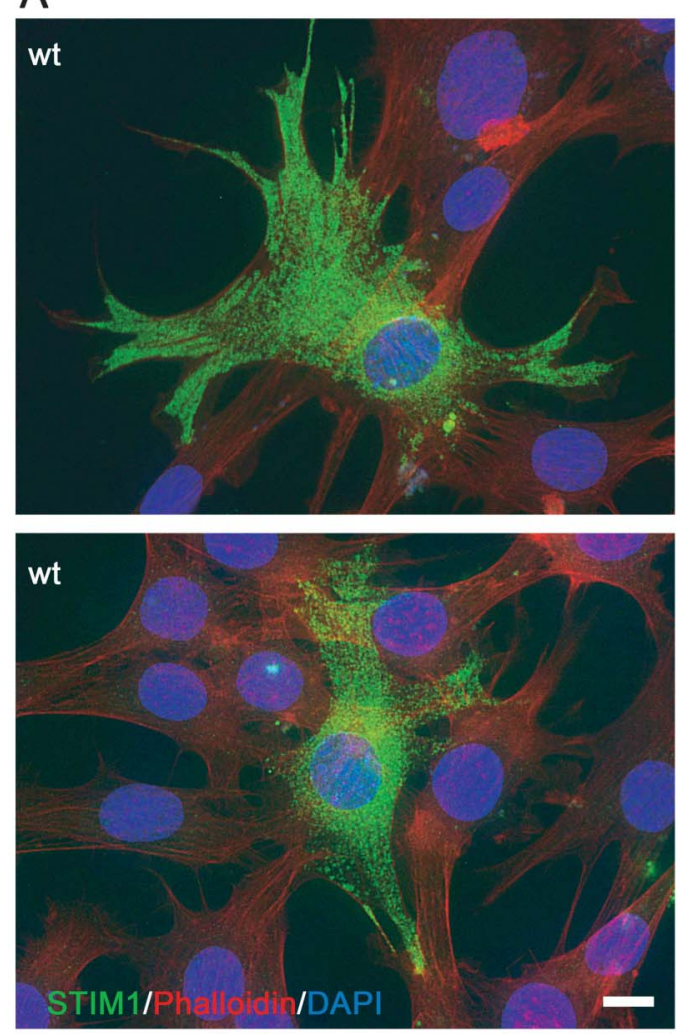

C

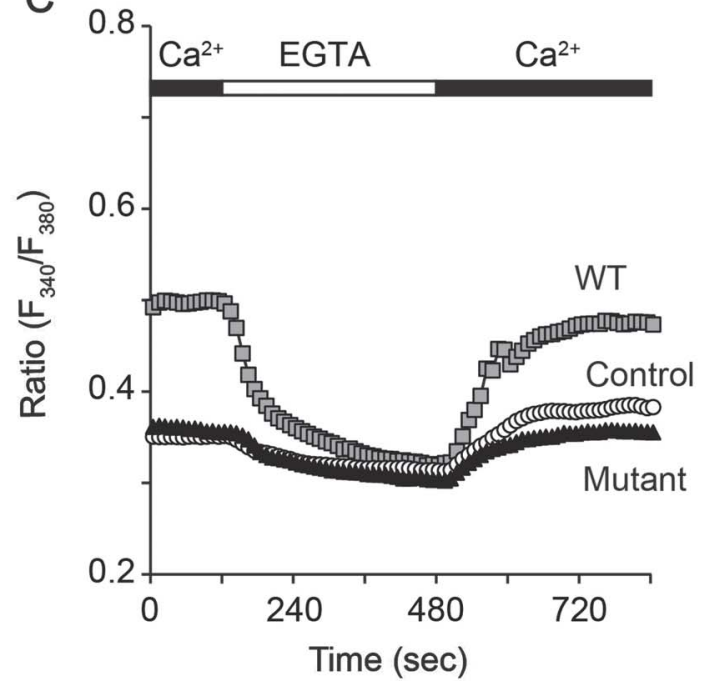

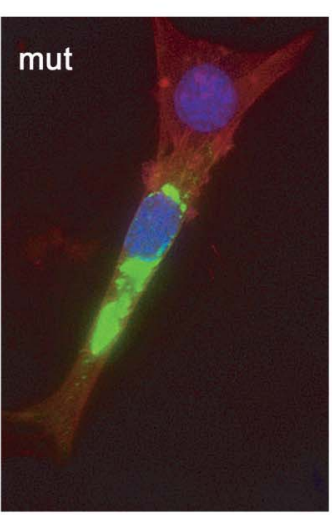

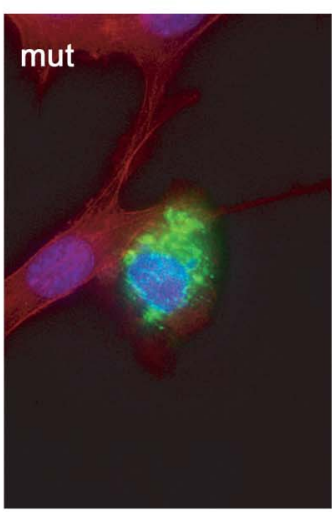

B

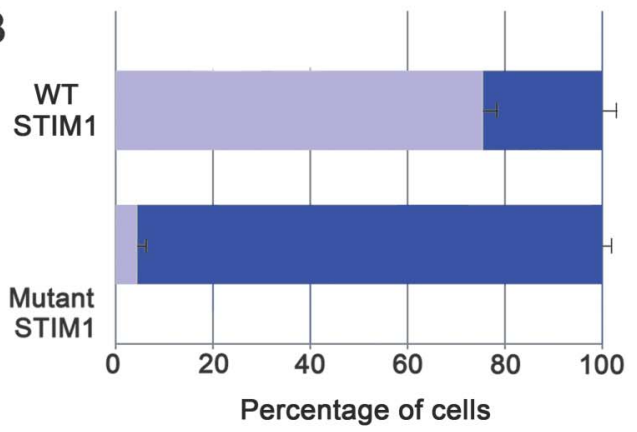

Diffuse localization

Aggregate-like localization

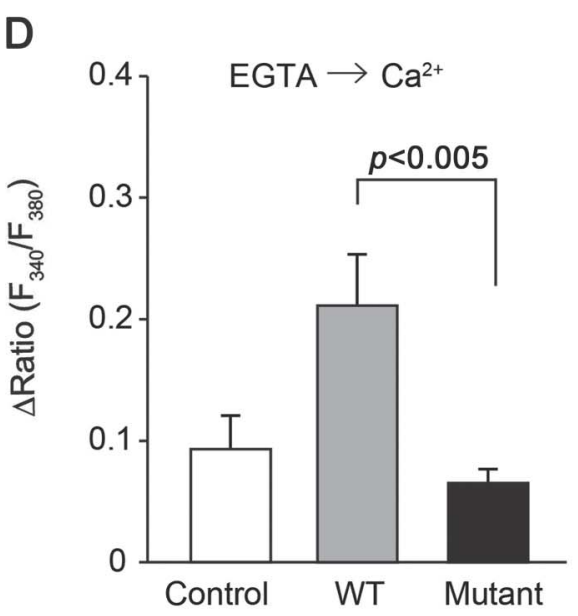

(A) C2C12 myoblasts were transfected with wild-type (WT) and the C-terminal inhibitory domain mutant STIM1 and labeled with anti-STIM1 antibody, phalloidin, and 4',6-diamidino-2-phenylindole (DAPI). WT STIM1 displays diffuse distribution in C2C12 cells, whereas the signal of the mutant STIM1 concentrates intensely around nuclei and exhibits an aggregation-like appearance in the shrunk cytoplasm. Bar $=50 \mu \mathrm{m}$. (B) Seven stitching images $(5 \times 5)$ of the C2C12 myoblasts were captured using a fluorescence microscope, and the number of cells with diffuse vs aggregation-like localization of STIM1 was counted. The majority of cells expressing WT STIM1 show diffuse localization, whereas most cells expressing the mutant STIM1 exhibit the aggregation-like pattern. (C) Intracellular $\mathrm{Ca}^{2+}$ concentration was measured in $\mathrm{HEK} 293$ cells expressing WT STIM1 (gray square; $n=40$ cells), the mutant STIM1 (black triangle; $n=42$ cells), or control (open circle; $n=$ 47 cells). The perfusion solution was changed from HEPES-buffered saline (HBS) containing $2 \mathrm{mM} \mathrm{Ca}^{2+}$ to $\mathrm{Ca}^{2+}$-free solution containing $0.5 \mathrm{mM}$ ethylene glycol tetraacetic acid (EGTA). To evaluate $\mathrm{Ca}^{2+}$ influx across the plasma membrane, $2 \mathrm{mM} \mathrm{Ca}^{2+}$ was applied to the cells. The duration of exposure to $\mathrm{Ca}^{2+}$-containing $\mathrm{HBS}$ and $\mathrm{Ca}^{2+}$-free $\mathrm{HBS}$ is indicated above the graph. (D) Maximal intracellular $\mathrm{Ca}^{2+}$ rises after readdition of $2 \mathrm{mM} \mathrm{Ca}^{2+}$ to the cells. Data points are mean $\pm \mathrm{SEM} . \mathrm{p}=$ $0.0028,0.0104$, and 0.5299 for WT vs mutant, control vs WT, and control vs mutant, respectively. 
Effects of STIM1 mutation on store-operated $\mathrm{Ca}^{2+}$ entrymediated $\mathrm{Ca}^{2+}$ influx. Finally, we performed $\mathrm{Ca}^{2+}$ measurements to examine whether the STIM1 mutation affects store-operated $\mathrm{Ca}^{2+}$ entry (SOCE). The wild-type or the mutant STIM1 was expressed in HEK293 cells, and changes in the intracellular $\mathrm{Ca}^{2+}$ concentration $\left(\left[\mathrm{Ca}^{2+}\right]_{\mathrm{i}}\right)$ were evaluated. As shown in figure 3C, overexpression of wild-type STIM1 caused an increased $\left[\mathrm{Ca}^{2+}\right]_{\mathrm{i}}$ in the perfusion solution containing $2 \mathrm{mM} \mathrm{Ca}^{2+}$, suggesting that wild-type STIM1 enhanced $\mathrm{Ca}^{2+}$ influx through ORAI1 endogenously expressed in HEK293 cells. This notion was further confirmed by the fact that removal of the external $\mathrm{Ca}^{2+}$ by ethylene glycol tetraacetic acid led to decreased $\left[\mathrm{Ca}^{2+}\right]_{\mathrm{i}}$ and that readdition of $\mathrm{Ca}^{2+}$ to the perfusion solution resumed the $\mathrm{Ca}^{2+}$ response. However, the mutant STIM1expressed cells did not show significant $\mathrm{Ca}^{2+}$ responses by the changes in the external $\mathrm{Ca}^{2+}$ concentration (figure 3, C and D). These results collectively suggest that the STIM1 mutation we found in this study causes dysregulated $\mathrm{Ca}^{2+}$ homeostasis.

DISCUSSION In the present study, we described a family with dominant TAM with a novel frameshift mutation in the CTID of STIM1. Of note, the triceps surae muscles were most severely involved in our patient. This is in contrast to the previous reports of TAM, in which the proximal muscles were predominantly affected. ${ }^{4,5}$ In skeletal muscle, $\mathrm{Ca}^{2+}$ homeostasis is regulated by SOCE. In SOCE, STIMI forms an oligomer upon the depletion of $\mathrm{Ca}^{2+}$ in SR and activates ORAI1 channels at sarcolemma, triggering extracellular $\mathrm{Ca}^{2+}$ entry. ${ }^{10}$ All STIM1 mutations in TAM reported so far are missense mutations residing in the EF hand (EF1 and EF2 in figure 2B), and this region is considered a hotspot. ${ }^{4-6}$ These mutations are presumed to induce the constitutive activation of ORAI1 and cause excessive $\mathrm{Ca}^{2+}$ influx into muscle cells.

In contrast, a frameshift mutation, which creates a premature stop codon in the CTID located in the Cterminal cytoplasmic region of STIM1, was identified in our family (figure 2B). The CTID is known to interact with SOCE-associated regulatory factor (SARAF), a regulator of slow $\mathrm{Ca}^{2+}$-dependent inactivation of $\mathrm{OR}$ AI1. ${ }^{11}$ Following the transfection of C2C12 myoblasts with the CTID mutant STIM1, aggregation-like signals of STIM1 were observed around the nuclei. The aggregation-like appearance is distinct from that in C2C12 myoblasts transfected with STIM1 harboring EF hand mutations, which exhibit numerous punctalike small clusters in cytoplasm. ${ }^{4,6}$

Furthermore, intracellular $\mathrm{Ca}^{2+}$ measurements revealed that the $\mathrm{Ca}^{2+}$ influx is significantly decreased in the cells transfected with the CTID mutant compared to the wild-type STIM1-transfected cells. This result is in marked contrast to the previous reports, in which increased $\mathrm{Ca}^{2+}$ influx caused by constitutive activation of SOCE was observed. ${ }^{4,7}$ The transcript of CTID mutant was present in the affected skeletal muscle of our patient, suggesting that nonsense-mediated mRNA decay is not induced. We speculate that the CTID mutant may inhibit the oligomer formation of STIM1 necessary to activate the ORAI1 channels, leading to the decreased intracellular $\mathrm{Ca}^{2+}$ influx. Alternatively, the STIM1 oligomer including the CTID mutant may not translocate properly to the subsarcolemma where the oligomer interacts with ORAI1, because the CTID mutant lacks the polybasic motif ( $\mathrm{K}$ in figure $2 \mathrm{C}$ ) necessary for the STIM1 targeting to plasma membrane. ${ }^{12}$ It is of particular interest to note that STIM2 $\beta$, an alternatively spliced form of STIM2, converts STIM isoforms from an activator to an inhibitor of ORAI1. ${ }^{13}$ Our findings provide further evidence that the dysregulation of $\mathrm{Ca}^{2+}$ homeostasis underlies the pathomechanism of TAM.

\section{AUTHOR CONTRIBUTIONS}

Dr. Okuma contributed to the evaluation of patients, data acquisition interpretation and analysis, and manuscript preparation. Dr. Saito contributed to the study concept and design, data acquisition, interpretation and analysis, and manuscript preparation. Dr. Mitsui, Dr. Hara, and Ms. Ikeda contributed to data acquisition, interpretation, and analysis. Dr. Hatanaka contributed to the evaluation of patients. Dr. T. Shimizu contributed to the study concept, design, and data analysis. Dr. Matsumura contributed to the study concept and design. Dr. J. Shimizu contributed to data acquisition, interpretation, and analysis. Dr. Tsuji contributed to the study concept and design. Dr. Sonoo contributed to the study concept and design, data acquisition, interpretation, and analysis.

\section{ACKNOWLEDGMENT}

The authors thank the patients and their families for participation in this study and Dr. Toshihiro Masaki and Dr. Hiroki Hagiwara (Teikyo University of Science) for useful discussion.

\section{STUDY FUNDING}

No targeted funding reported.

\section{DISCLOSURE}

Dr. Okuma reports no disclosures. Dr. Saito has received travel funding/ speaker honoraria from Eisai Co. Ltd., Takeda Pharmaceutical Co. Ltd., Daiichi Sankyo Co. Ltd., and Otsuka Pharmaceutical Co. Ltd.; has received research support from Eisai Co. Ltd., Takeda Pharmaceutical Co. Ltd., and Daiichi Sankyo Co. Ltd.; and was supported by an Intramural Research Grant (26-8) for Neurological and Psychiatric Disorders of NCNP from the Ministry of Health, Labour and Welfare of Japan and a Grant-in-Aid for Scientific Research C (26461281) from the Ministry of Education, Culture, Sports, Science and Technology of Japan. Dr. Mitsui has received research support from the Ministry of Education, Culture, Sports, Science and Technology of Japan (Grant-in-Aid for Scientific Research (C) 15K09334). Dr. Hara was supported by an Intramural Research Grant (26-8) for Neurological and Psychiatric Disorders of NCNP from the Ministry of Health, Labour and Welfare of Japan, JSPS KAKENHI Grant Number 15H04846, and a Grant-in-Aid for Challenging Exploratory Research (25670149, 15K13741) from The Ministry of Education, Culture, Sports, Science and Technology, Japan; and has received research support from the Takeda Science Foundation, the Life 
Science Foundation of Japan, the Kowa Life Science Foundation, and the Salt Science Research Foundation. Dr. Hatanaka and Ms. Ikeda report no disclosures. Dr. T. Shimizu was supported by a Grant-in-Aid for Scientific Research C (15K09328) from the Ministry of Education, Culture, Sports, Science and Technology of Japan. Dr. Matsumura was supported by a Grant-in-Aid for Scientific Research C (25430075) from the Ministry of Education, Culture, Sports, Science and Technology of Japan. Dr. J. Shimizu was supported by a Health and Labour Sciences Research Grant on Intractable Diseases (Evidence-based Early Diagnosis and Treatment Strategies for Neuroimmunological Diseases) from the Ministry of Health, Labour and Welfare of Japan, a Grant-in-Aid for Scientific Research C (24591289) from the Ministry of Education, Culture, Sports, Science and Technology of Japan, and an Intramural Research Grant (268) for Neurological and Psychiatric Disorders of NCNP from the Ministry of Health, Labour and Welfare of Japan. Dr. Tsuji has received travel/ speaker honoraria from Kowa Pharmaceutical Co. Ltd., Japan Blood Products Organizations, Mitsubishi Tanabe Pharma Co., Bayer Japan, Janssen Pharmaceutical, Genzyme Japan, Eisai Co. Ltd., FPPharmaceutical Co., Dainippon Sumitomo Pharma Co. Ltd., Otsuka Pharmaceutical Co. Ltd., Actelion Pharmaceuticals Japan Ltd., Sony Corporation, Nikkei Business Publications Inc., and Leave a Nest Co. Ltd.; has served on the editorial board of Neurology and Clinical Neuroscience; has received research support from Sanofi, Japan Blood Products Organization, Mitsubishi Tanabe Pharma Co., Pfizer Japan Inc., Ono Pharmaceutical Co. Ltd., Daiichi Sankyo Co. Ltd., Eisai Co. Ltd., Kowa Pharmaceutical Co. Ltd., and GlaxoSmithKline; and was supported by KAKENHI (Grant-in-Aid for Scientific Research on Innovative Areas [22129001 and 22129002]), the Japan Agency for Medical Research and Development (Grant-in-Aid [H26-Nanchiseishikkan JitsuyokaIppan-080, 26310101, and Ninchisho Kenkyu 26340101]), and a Grant-in-Aid (H23-Jitsuyoka [Nanbyo]-Ippan-004) of the Research on Measures for Intractable Diseases from the Ministry of Health, Welfare and Labour, Japan. Dr. Sonoo has served on the editorial boards of Muscle and Nerve, Clinical Neurology, and the Japanese Journal of Clinical Neurophysiology; and was supported by Grants-in-Aid for Scientific Research from the Ministry of Education, Science, Sports and Culture of Japan (23591285), and a Health and Labour Sciences Research Grant on Rare and Intractable Diseases (Evidence-based Early Diagnosis and Treatment Strategies for Neuroimmunological Diseases) from the Ministry of Health, Labour and Welfare of Japan. Go to Neurology.org/ng for full disclosure forms.

Received March 17, 2015. Accepted in final form December 7, 2015.

\section{REFERENCES}

1. Engel WK, Bishop DW, Cunningham GG. Tubular aggregates in type II muscle fibers: ultrastructural and histochemical correlation. J Ultrastruct Res 1970;31:507-525.
2. Schiaffino S. Tubular aggregates in skeletal muscle: just a special type of protein aggregates? Neuromuscul Disord 2012;22:199-207.

3. Pavlovicová M, Novotová M, Zahradník I. Structure and composition of tubular aggregates of skeletal muscle fibres. Gen Physiol Biophys 2003;22:425-440.

4. Böhm J, Chevessier F, Maues De Paula A, et al. Constitutive activation of the calcium sensor STIM1 causes tubular-aggregate myopathy. Am J Hum Genet 2013;92: 271-278

5. Hedberg C, Niceta M, Fattori F, et al. Childhood onset tubular aggregate myopathy associated with de novo STIM1 mutations. J Neurol 2014;261:870-876.

6. Böhm J, Chevessier F, Koch C, et al. Clinical, histological and genetic characterisation of patients with tubular aggregate myopathy caused by mutations in STIM1. J Med Genet 2014;51:824-833.

7. Endo Y, Noguchi S, Hara Y, et al. Dominant mutations in ORAI1 cause tubular aggregate myopathy with hypocalcemia via constitutive activation of store-operated $\mathrm{Ca}^{2+}$ channels. Hum Mol Genet 2015;24:637-648.

8. Nesin V, Wiley G, Kousi M, et al. Activating mutations in STIM1 and ORAI1 cause overlapping syndromes of tubular myopathy and congenital miosis. Proc Natl Acad Sci U S A 2014;111:4197-4202.

9. Picard C, McCarl CA, Papolos A, et al. STIM1 mutation associated with a syndrome of immunodeficiency and autoimmunity. N Engl J Med 2009;360:1971-1980.

10. Stiber J, Hawkins A, Zhang ZS, et al. STIM1 signaling controls store-operated calcium entry required for development and contractile function in skeletal muscle. Nat Cell Biol 2008;10:688-697.

11. Jha A, Ahuja M, Maléth J, et al. The STIM1 CTID domain determines access of SARAF to SOAR to regulate Orail channel function. J Cell Biol 2013;202:71-79.

12. Liou J, Fivaz $M$, Inoue $T$, et al. Live-cell imaging reveals sequential oligomerization and local plasma membrane targeting of stromal interaction molecule 1 after $\mathrm{Ca}^{2+}$ store depletion. Proc Natl Acad Sci U S A 2007;104: 9301-9306.

13. Rana A, Yen M, Sadaghiani AM, et al. Alternative splicing converts STIM2 from an activator to an inhibitor of store-operated calcium channels. J Cell Biol 2015;209: 653-669. 


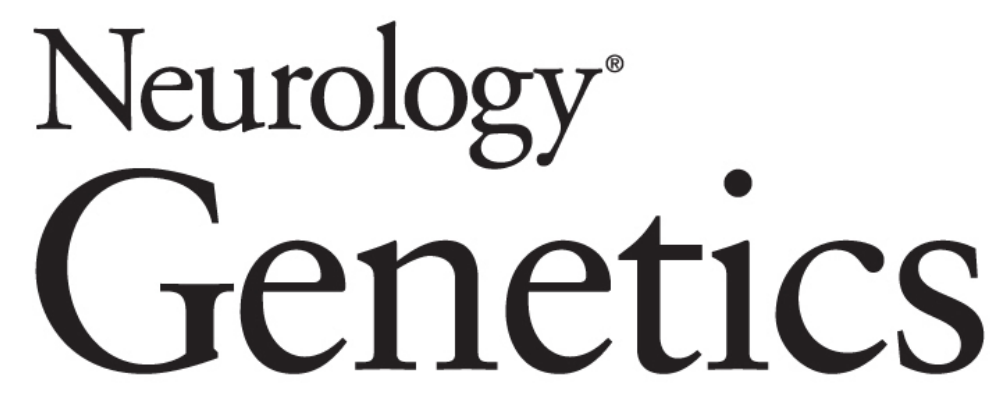

Tubular aggregate myopathy caused by a novel mutation in the cytoplasmic domain of STIM1

Hidehiko Okuma, Fumiaki Saito, Jun Mitsui, et al.

Neurol Genet 2016;2;

DOI 10.1212/NXG.0000000000000050

This information is current as of February 1, 2016

\section{Updated Information \&}

Services

References

Citations

Subspecialty Collections

Permissions \& Licensing

Reprints including high resolution figures, can be found at: http://ng.neurology.org/content/2/1/e50.full.html

This article cites 13 articles, 5 of which you can access for free at: http://ng.neurology.org/content/2/1/e50.full.html\#\#ref-list-1

This article has been cited by 2 HighWire-hosted articles: http://ng.neurology.org/content/2/1/e50.full.html\#\#otherarticles

This article, along with others on similar topics, appears in the following collection(s):

\section{All Genetics}

http://ng.neurology.org//cgi/collection/all_genetics

Muscle disease

http://ng.neurology.org//cgi/collection/muscle_disease

Information about reproducing this article in parts (figures,tables) or in its entirety can be found online at:

http://ng.neurology.org/misc/about.xhtml\#permissions

Information about ordering reprints can be found online:

http://ng.neurology.org/misc/addir.xhtml\#reprintsus

Neurol Genet is an official journal of the American Academy of Neurology. Published since April 2015, it is an open-access, online-only, continuous publication journal. Copyright $\odot 2016$ American Academy of Neurology. All rights reserved. Online ISSN: 2376-7839.

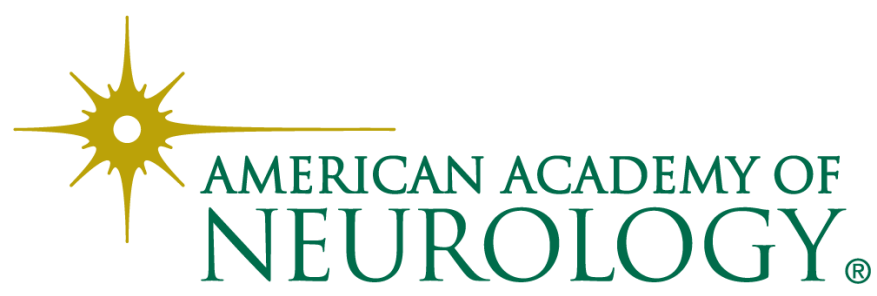

